\title{
REGRESSÃO SEGMENTADA COMO MODELO NA DETERMINAÇÃO DE EXIGÊNCIAS NUTRICIONAIS DE PEIXES
}

\author{
Leandro Portz'; Carlos Tadeu dos Santos Dias²; José Eurico Possebon Cyrino ${ }^{3 \star}$ \\ ${ }^{1}$ Pós-Graduando do Depto. de Produção Animal - USP/ESALQ. \\ ${ }_{3}^{2}$ Depto. de Ciências Exatas - USP/ESALQ. \\ ${ }^{3}$ Depto. de Produção Animal - USP/ESALQ, C.P. 9 - CEP: 13418-900 - Piracicaba, SP. \\ *Autor correspondente <jepcyrin@carpa.ciagri.usp.br>
}

RESUMO: A produção de peixes como atividade agroindustrial vem crescendo consideravelmente, motivando o crescimento das indústrias especializadas em produção de rações para peixes e crustáceos e criando grande demanda por conhecimentos específicos sobre as exigências nutricionais das várias espécies de peixes e crustáceos utilizados em aquicultura comercial. As exigências nutricionais dos organismos aquáticos podem ser influenciadas por vários fatores, incluindo tamanho do peixe, composição e forma da dieta, manejo alimentar, sistema e regime de produção. Além disso os tipos de modelos estatísticos e matemáticos utilizados na determinação destas exigências podem ou não ser adequados à estimativa dos parâmetros biológicos avaliados. O presente trabalho apresenta uma avaliação crítica da adequação do modelo "broken-line" na análise de resultados de um experimento de exigências nutricionais em proteína para o tambaqui. Os resultados permitem inferir que a aplicação do modelo "brokenline analysis", feito pelo procedimento PROC NLIN do SAS, é um método de análise simples, rápido e eficiente na determinação de exigências nutricionais, que resultam de dados tomados em experimentos de ganho de peso para peixes. O modelo poderia, entretanto, subestimar valores determinados por outros modelos tradicionalmente utilizados que, mesmo assim, podem não ser, necessariamente, mais precisos.

Palavras-chave: "broken-line", exigência nutricional, peixe

\section{A BROKEN-LINE MODEL TO FIT FISH NUTRITION REQUIREMENTS}

\begin{abstract}
The growth of the fish industry, as a result of the development of aquaculture, requires specific knowledge on nutritional requirements of cultured fish and shellfish species. These requirements can be influenced by factors as diverse as fish size, feed composition, feeding practices, system management, as well as by the statistical and mathematical tools utilized in the analysis of fish nutritional research data. This paper presents a critical appraisal on the use of the 'broken-line' model in the analysis of growth data derived from a dietary protein requirement assay with 'tambaqui' (Colossoma macropomun). Results show that the 'broken-line model analysis', as performed by a linear regression procedure, is simple, fast and reliable, suitable for the analysis of fish growth data. Nevertheless, the model may underestimate values determined by other methods, which may or not have higher precision levels.

Key words: 'broken line analysis', nutritional requirement, fish
\end{abstract}

\section{INTRODUÇÃO}

Durante as últimas décadas a produção de peixes como atividade agroindustrial cresceu consideravelmente, motivando o crescimento das indústrias especializadas em produção de rações para peixes e crustáceos. Entretanto, pouco ainda é conhecido das exigências qualitativas e quantitativas de nutrientes das várias espécies nacionais utilizadas em aquicultura, em comparação ao acervo técnico-científico conhecido para as espécies exóticas (Anderson et al., 1981; Steffens, 1989; Wilson, 1989; Cho, 1992; Cowey, 1992; Castagnolli, 1979; Pezzato, 1997; Carneiro et al., 1990). Por esta ração, existem no mercado um número reduzido de rações consideradas nutricionalmente completas para as espécies indígenas.

O conhecimento das exigências nutricionais dos peixes é de vital importância para a produtividade e economicidade dos sistemas de produção, e aproveitamento eficiente dos nutrientes das dietas. O ajuste espécie específico das exigências nutricionais e o emprego de técnicas avançadas de cocção e expansão na fabricação das rações, aumenta a biodisponibilidade de nutrientes, melhorando assim a assimilação pelos peixes (Kiang, 1998).

As exigências nutricionais dos peixes podem ser influenciadas por vários fatores, incluindo tamanho do peixe, composição e forma da dieta, manejo alimentar, sistema e regime de produção, além dos tipos de modelos estatísticos e matemáticos 
utilizados na determinação destas exigências, que podem ou não ser adequados à estimativa dos parâmetros avaliados. Gatlin (1998) cita que normalmente são estabelecidos parâmetros de confiança em exigências nutricionais para as espécies em produção nos diversos sistemas aquaculturais, onde as estimativas nutricionais são baseadas no hábito alimentar natural das espécies.

A determinação de exigências nutricionais para peixes não depende apenas da interpretação estatística dos resultados experimentais. A maior ou menor precisão dos resultados vai depender da escolha de um modelo matemático que possua como critério de avaliação diferentes respostas às concentrações de nutrientes testados nas dietas.

A escolha de um modelo apropriado para descrever respostas à variação de parâmetros nutricionais em peixes já foi discutida por vários autores, entre eles Zeitoun et al. (1976), Mercer (1982), Schultz (1987) e Robbins et al. (1979). Quando tratamos de níveis de exigência de um determinado nutriente na dieta de uma espécie, o modelo de regressão segmentada - modelo "brokenline" ou "Linear Response Plateau - LRP" ou modelo descontínuo - permite resultados mais coerentes em uma avaliação final, em comparação com outros modelos não lineares, os quais poderiam estar superestimando os valores encontrados. O modelo da regressão segmentada ou modelo descontínuo não é muito entendido e sua descrição não generalizada é pouco usada em trabalhos de exigências nutricionais para peixes no Brasil (Euclides, 1983). Contudo, este modelo é considerado como simples e de fácil interpretação (Robbins, 1986).

O desbalanço de qualquer nutriente em uma ração completa para peixes pode trazer sérios riscos ao sistema de produção e problemas de distúrbios nutricionais, muito comuns em sistemas de criação intensiva (Tacon, 1992). Portanto, quando se determinam exigências nutricionais para peixes objetiva-se, teoricamente, alcançar o máximo potencial biológico da espécie em estudo. Isto torna necessário o uso de técnicas estatísticas adequadas e modelos matemáticos exatos para determinação dos parâmetros biológicos da espécie.

A proposta de descrever este modelo baseiase então na necessidade de determinar com precisão resultados de experimentos dose-resposta de níveis de exigências nutricionais em dietas para peixes, ou seja, determinar o nível mínimo de um nutriente que garanta o máximo desempenho de uma espécie. Esta resposta é considerada um importante determinante da relação custo-benefício na composição de rações para peixes, uma vez que alguns ingredientes são considerados fatores economicamente limitantes nas formulações.
O ganho de peso é a variável quantitativa mais comumente utilizada em experimentos de avaliação de dietas com diferentes níveis de um determinado nutriente essencial para peixes (Robbins et al., 1979). O fenômeno do crescimento está relacionado ao aumento de peso corporal aliado ao desenvolvimento progressivo do peixe. Assim pode-se dizer que 0 ganho de peso é o melhor parâmetro utilizado para avaliação da adequação nutricional de uma dieta ou da exigência nutricional de uma espécie. Normalmente, a utilização do parâmetro de ganho de peso na avaliação quantitativa das exigências nutricionais para peixes é condicionada à existência de condições experimentais controladas, onde o crescimento do peixe pode ser prejudicado ou não somente se algum nutriente essencial for inadequadamente suprido ou retirado da dieta.

O modelo da regressão segmentada tem sido utilizado na análise de resultados de experimentos de exigências nutricionais em proteína, aminoácidos essenciais, vitaminas e minerais. Zeitoun et al. (1976) determinaram a exigência de proteína dietética para a truta arco-íris (Oncorhynchus mykiss) a partir da análise da curva de ganho de peso da espécie por este modelo matemático. Mahajan \& Agrawal (1980) utilizaram este modelo para avaliar exigências em ácido ascórbico para carpa indiana (Cirrhina mrigala). O modelo da regressão segmentada foi ainda considerado ideal na avaliação das exigências nutricionais em proteína de alevinos e juvenis do "bass" (Micropterus dolomieui e Micropterus salmoides) (Anderson et al., 1981), e na avaliação das exigências em aminoácidos do "striped bass" híbrido (Griffin et al., 1994; Keembiyehetty \& Gatlin III, 1993).

Finalmente, o modelo da regressão segmentada também já foi utilizado na avaliação de experimentos de determinação de exigências em proteína de espécies de peixes marinhos (Moore et al., 1988), e na quantificação das exigências em vitamina K (Shiau \& Liu, 1994), niacina (Shiau \& Suen, 1994) e vitamina C (Shiau, 1994) para crustáceos, em especial para o camarão marinho (Penaeus monodon). A expansão da indústria americana do bagre do canal (Ictalurus punctatus) e da produção comercial da tilápia do Nilo (Oreochromis niloticus), condicionou a realização de pesquisas em exigências nutricionais para estas espécies, e aí também o método da regressão segmentada foi utilizado na avaliação dos resultados de experimentos de determinação em exigências com ácido fólico, lisina e arginina para o bagre do canal, bem como de niacina, lisina, arginina, histidina, valina, leucina, isoleucina, treonina, triptofano, metionina e fenilalanina para a tilápia nilótica (Robinson et al., 1981; Duncan et al., 1993; Shiau \& Suen, 1992; Santiago \& Lovell, 1988). 


\section{A REGRESSÃO SEGMENTADA COMO MODELO}

Possivelmente a primeira investigação utilizando o modelo "broken line" em experimentos com peixes tenha sido conduzida com o salmão (Oncorhynchus tshawytscha) por De Long et al. (1958), tendo por base técnicas pioneiras desenvolvidas para determinação das exigências nutricionais de animais terrestres. Posteriormente, Zeitoun et al. (1976) trabalhando com truta arco-íris (Oncorhynchus mykiss), cita este modelo utilizado por $\mathrm{H}$. W. Norton da Universidade de Illinois (Urbana) em 1971, em trabalhos que determinavam aspectos quantitativos da exigência de $D$ e L-triptofano em dietas para leitões (Baker et al., 1971).

Mameesh et al. (1956) citam que o ajuste do modelo matemático da regressão segmentada é feito pelo método dos mínimos quadrados, e objetiva estimar exigências de um determinado nutriente dosado em uma dieta. O modelo propõe basicamente a existência de uma relação linear positiva de crescimento (ganho de peso) no eixo "Y" de um gráfico, em comparação a níveis dietéticos de um nutriente essencial (eixo "X"), onde é determinado o chamado "break-point" - ponto de quebra, ou seja: o ponto que representa a menor soma dos quadrados dos desvios. O ponto de quebra é considerado como o nível ideal de um nutriente necessário para o máximo ganho de peso de uma espécie (Figura 1).

Ainda nesta figura, para o nível mínimo em exigência protéica (35\%), a letra "R" representa o ponto de mudança de inclinação da reta ("quebra") da linha ascendente para horizontal, no instante em que o animal apresenta crescimento ótimo no nível mínimo necessário de proteína. Todos os valores do eixo " $X$ " acima deste ponto são ajustados em uma equação segundo um modelo de regressão linear onde o componente funcional (ou matemático) é do tipo $\mathrm{y}=$ $a+b x$. No desenvolvimento do modelo são feitas tentativas de várias regressões usando diferentes pontos

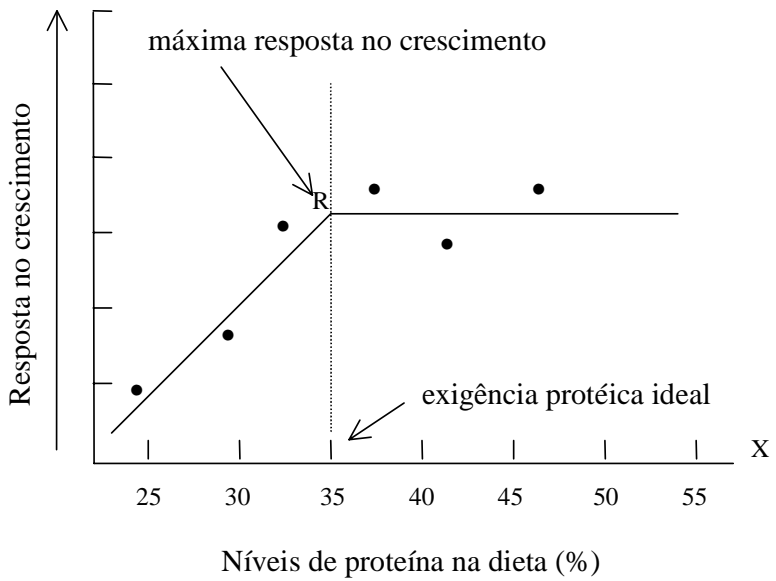

Figura 1 - Ilustração gráfica do uso do modelo de regressão segmentada. de mudança de inclinação da reta, onde a soma de quadrado dos desvios de cada regressão é obtida.

A regressão linear que fornecer o menor erro da soma do quadrado médio é considerada como melhor grau de inclinação da reta que representa a linearidade entre os níveis de doses estimados, cruzando com a resposta em ganho de peso.

\section{DESCRIÇÃO E EXEMPLO PRÁTICO DO MODELO}

Segundo Robbins (1986) o modelo de regressão segmentada consiste em duas partes: uma linha inclinada ascendente ou descendente seguida de uma linha horizontal, onde seus pontos de interseção vão determinar o ponto de quebra. Este modelo de uma inclinação é mais adequado para estimar parâmetros de crescimento. Para outros tipos de variáveis biológicas, a equação do modelo de regressão segmentada descreve duas linhas de intersecção, ambas com inclinação diferente a zero.

Segundo Robins (1986), o modelo de regressão utilizado é do tipo:

$$
Y_{\mathrm{i}}=L+U\left(R-X_{\mathrm{LRi}}\right)+\mathrm{e}_{\mathrm{i}}, i=1,2 \ldots n_{1}, n_{1+1}, \ldots, n
$$

cujo $\left(R-X_{\mathrm{LRi}}\right)=0$ para $i \geq n_{1}+1$, e $n_{1}$ é o número de observações até o ponto de quebra, e $n$ é o número de pares de observações.

Este mesmo autor descreve outro modelo de regressão utilizado como sendo do tipo:

$$
Y_{\mathrm{i}}=L+U\left(R-X_{\mathrm{LRi}}\right)+V\left(X_{\mathrm{GRi}}-R\right)
$$

cujo $\left(R-X_{\mathrm{LRi}}\right)=0$ para $i>n_{1}$ e $\left(X_{\mathrm{GRi}}-R\right)=0$ para $i \leq n_{1}$.

Nestes modelos, $L$ representa a coordenada no eixo das ordenadas e $R$ no eixo das abcissas de um ponto de quebra (ponto de quebra) em uma curva. $U$ é o coeficiente de inclinação de uma linha quando $X<R$, e no modelo de duas inclinações o $V$ representa a inclinação de uma linha quando $X>R$ e e é o componente aleatório ou resíduo. Assim pela definição $\left(R-X_{L R}\right)$ é zero quando $X>R$ e $\left(X_{G R}-R\right)$ é zero quando $X \leq R$. No sistema computacional SAS, o modelo normalmente é ajustado pela determinação da menor soma de quadrados médios. Utilizando-se da anotação matricial os modelos anteriormente mencionados podem ser expressos:

1. Modelo de uma inclinação:

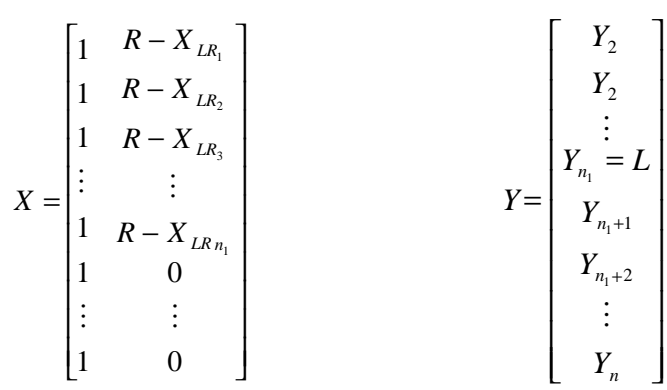


$X^{\prime} X=\left[\begin{array}{cc}n & \sum_{i=1}^{n}\left(R-X_{L R_{i}}\right) \\ \sum_{i=1}^{n}\left(R-X_{L R_{i}}\right) & \sum_{i=1}^{n}\left(R-X_{L R_{i}}\right)^{2}\end{array}\right] \quad X^{\prime} Y=\left[\begin{array}{c}\sum_{i=1}^{i} Y_{i} \\ \sum_{i=1}^{n}\left(R-X_{L R_{i}}\right) Y_{i}\end{array}\right]$

Onde: $\left(X^{\prime} X\right)^{-1} X^{\prime} Y=B=\left[\begin{array}{c}L \\ U\end{array}\right]$

2. Modelo de duas inclinações:

$X=\left[\begin{array}{ccc}1 & R-X_{L R} & 0 \\ 1 & R-X_{L R} & 0 \\ 1 & R-X_{L R} & 0 \\ 1 & R-X_{L R} & 0 \\ \cdots & \cdots & \cdots \\ 1 & 0 & X_{G R}-R \\ \vdots & \vdots & X_{G R}-R \\ 1 & 0 & X_{G R}-R\end{array}\right] \quad Y=\left[\begin{array}{c}Y_{2} \\ Y_{2} \\ \vdots \\ Y_{n_{1}}=L \\ Y_{n_{1}}+1 \\ Y_{n_{1}}+2 \\ \vdots \\ Y_{n}\end{array}\right]$

$X^{\prime} X=\left[\begin{array}{ccc}n & \sum\left(R-X_{L R}\right) & \sum\left(X_{G R}-R\right) \\ \sum\left(R-X_{L R}\right) & \sum\left(R-X_{L R}\right)^{2} & 0 \\ \sum\left(X_{G R}-R\right) & 0 & \sum\left(X_{G R}-R\right)^{2}\end{array}\right] X^{\prime} Y\left[\begin{array}{c}\sum Y \\ \sum\left(R-X_{L R}\right) Y \\ \sum\left(X_{G R}-R\right) Y\end{array}\right]$

A soma de quadrados do modelo é igual a $B^{\prime} X^{\prime} Y$. Usando um processo iterativo, podemos obter a estimativa de máxima verossimilhança de $R$, isto é, o valor de $R$ que maximiza a soma de quadrados do modelo. As variâncias amostrais aproximadas de $L, U$, $V$ e $R$ podem ser estimadas da matriz de somas de quadrados e produtos das primeiras derivadas. Estas matrizes podem ser descritas como:

1. Modelo de uma inclinação:

$D^{\prime} D=\left[\begin{array}{ccc}n & \sum\left(R-X_{L R}\right) & n_{L R} U \\ \sum\left(R-X_{L R}\right) & \sum\left(R-X_{L R}\right)^{2} & U \sum\left(R-X_{L R}\right) \\ n_{L R} U & U \sum\left(R-X_{L R}\right) & n_{L R} U^{2}\end{array}\right]$

2. Modelo de duas inclinações:

$D^{\prime} D=\left[\begin{array}{cccc}n & \sum\left(R-X_{L R}\right) & \sum\left(X_{G R}-R\right) & n_{L R} U-n_{G R} V \\ \sum\left(R-X_{L R}\right) & \sum\left(R-X_{L R}\right)^{2} & 0 & U \sum\left(R-X_{L L}\right) \\ \sum\left(X_{G R}-R\right) & 0 & \sum\left(X_{G R}-R\right)^{2} & -V \sum\left(X_{G R}-R\right) \\ n_{L R} U-V n_{G R} & U \sum\left(R-X_{L R}\right) & -V \sum\left(X_{G R}-R\right) & n_{L R} U^{2}+n_{G R} V^{2}\end{array}\right]$

Nestas matrizes, $n_{\mathrm{LR}}$ é o número de doses ou níveis com valores menores que $R$, e $n_{G R}$ é o número de doses com valores maiores que $R$. A matriz de covariâncias é obtida pelo produto do inverso da matriz $D^{\prime} D$ com seu erro apropriado, isto é, o quadrado médio do resíduo.

$$
\hat{\sum}=\left(d^{\prime} d\right)^{-1} \cdot Q M \text { Resíduo }
$$

Uma outra alternativa mais eficiente no ajuste do modelo "broken-line" é ajustar iterativamente um conjunto inicial de estimativas de parâmetros até que 0 vetor de ajuste seja próximo de zero. Para o modelo observamos os valores de $X\left(X_{1} \Lambda X_{\mathrm{m}} \Lambda X_{\mathrm{n}}\right), Y\left(Y_{1} \Lambda Y_{\mathrm{m}} \Lambda\right.$ $Y_{\mathrm{n}}$ ) e um vetor inicial de estimativas dos parâmetros,

$Q_{1}=\left[\begin{array}{l}L_{1} \\ U_{1} \\ R_{1}\end{array}\right]$, onde a j-ésima iteração é:

$D_{j}=\left[\begin{array}{ccc}1 & \left(R_{j}-X_{1}\right) & U_{j} \\ \vdots & \vdots & \vdots \\ 1 & \left(R_{j}-X_{m}\right) & U_{j} \\ \vdots & \vdots & \vdots \\ 1 & \left(R_{j}-X_{n}\right) & U_{j}\end{array}\right] \quad Y-Y_{j}=\left[\begin{array}{c}Y_{1}-Y_{X_{1}} \\ \vdots \\ Y_{m}-Y_{X_{m}} \\ \vdots \\ Y_{n}-Y_{X_{n}}\end{array}\right]$

$\left(D_{j}^{\prime} D_{j}\right)^{-1} D_{j}^{\prime}\left(Y-Y_{j}\right)=B_{j+1}=\left[\begin{array}{c}L_{j+1}-L_{j} \\ U_{j+1}-U_{j} \\ R_{j+1}-R_{j}\end{array}\right]$ (vetor de ajustes)

$Q_{j}+B_{j+1}=Q_{j+1}=\left[\begin{array}{c}L_{j+1} \\ U_{j+1} \\ R_{j+1}\end{array}\right]$

Similarmente, para o modelo de duas inclinações:

$D_{j}=\left[\begin{array}{cccc}1 & \left(R_{j}-X_{l}\right) & \left(X_{l}-R_{j}\right) & U_{j}-V_{j} \\ \vdots & \vdots & \vdots & \vdots \\ 1 & \left(R_{j}-X_{m}\right) & \left(X_{m}-R_{j}\right) & U_{j}-V_{j} \\ \vdots & \vdots & \vdots & \vdots \\ 1 & \left(R_{j}-X_{n}\right) & \left(X_{n}-R_{j}\right) & U_{j}-V_{j}\end{array}\right]$

$Y-Y_{j}=\left[\begin{array}{c}Y_{l}-Y_{X_{l}} \\ \vdots \\ Y_{m}-Y_{X_{m}} \\ \vdots \\ Y_{n}-Y_{X_{n}}\end{array}\right]$

$\left(D_{j}^{\prime} D_{j}\right)^{-1} D_{j}^{\prime}\left(Y-Y_{j}\right)=B_{j+1}=\left[\begin{array}{c}L_{j+1}-L_{j} \\ U_{j+1}-U_{j} \\ V_{j+1}-V_{j} \\ R_{j+1}-R_{j}\end{array}\right]$, (vetor de ajustes)

$Q_{j}+B_{j+1}=Q_{J+1}=\left[\begin{array}{c}L_{j+1} \\ U_{j+1} \\ V_{j+1} \\ R_{j+1}\end{array}\right]$ 
Acima, $Y_{j}$ é o valor de $Y$ estimado para um observado valor de $X$ usando as estimativas de parâmetros calculada na $J-1$ iteração.

Como exemplo prático serão utilizados os resultados da TABELA 1 determinados por Vidal et al. (1998), em estudo que visava determinar o nível de proteína dietética ideal para a espécie tambaqui (Colossoma macropomum) em experimentos realizados na Estação de Hidrobiologia da Universidade Federal de Viçosa. Para efeito de comparação com métodos tradicionais, como o de regressão quadrática utilizado pelo autor, será feita uma plotagem dos resultados de ganho de peso encontrado pelo autor utilizando o sistema computacional SAS. O método de ajuste iterativo de um conjunto de parâmetros estimados é baseado nos procedimentos utilizados pelo SAS (1985), através do PROC NLIN. O exemplo prático de programação é apresentado na TABELA 2.

A partir dos resultados processados obtémse a equação para o modelo de duas inclinações: $Y=204,84-14,82\left(21,08-X_{L R}\right)-1,86\left(X_{G R}-21,08\right) \quad$ e para 0 modelo de uma inclinação: $Y=196,32-14,82\left(20,5-X_{L R}\right)$. O modelo de uma inclinação seria o mais indicado para descrever o método proposto.

Analisando 0 efeito $(P<0,10$ - efeito quadrático) dos níveis de proteína bruta sobre o ganho de peso dos tambaquis, Vidal et al. (1998) observaram que a exigência nutricional em proteína para o tambaqui pode ser representada pela equação: $Y=-291,838+39,6419 X-0,792415 X^{2}$ cujo $X$ é o nível protéico e $Y$ o ganho de peso (modelo quadrático), que apresenta um ponto de ótimo $25,01 \%$. Os resultados obtidos do processamento do programa são mostrados na TABELA 3.

Para o modelo de regressão segmentada, $R^{2}$ foi estimado em $99,92 \%$ e a exigência em proteína no "break-point" em $20,05 \pm 0,512$. O ganho de peso neste ponto foi de 196,32g (Figura 2). Pode-se então concluir que o nível protéico ideal de $25,01 \%$, estimado por Vidal et al. (1998), poderia estar superestimado, já que 0 experimento foi feito com a espécie em uma faixa de peso de 30 a 250 gramas (juvenil e crescimento) e o ideal seria que o experimento fosse feito em uma fase com uma menor amplitude de limites de peso. Em adição, os autores relatam que havia uma produção média de $248 \mathrm{mg} \mathrm{m}^{-3}$ de fitoplâncton no ambiente, que pode ter contribuído significativamente na dieta da espécie. Se esta contribuição do alimento natural tivesse sido levada em conta na determinação da exigência nutricional, o modelo proposto poderia estar melhor adequado.
TABELA 1 - Desempenho de tambaquis de 30 a $250 \mathrm{~g}$, alimentados com rações contendo níveis crescentes de proteína bruta (Vidal et al., 1998).

\begin{tabular}{lcccccc}
\hline Parâmetro & \multicolumn{6}{c}{ Proteína bruta } \\
\cline { 2 - 6 } & 18 & 21 & 24 & 27 & 30 & CV(\%) \\
\hline $\begin{array}{l}\text { Ganho médio } \\
\text { de peso (g) }\end{array}$ & 159,26 & 203,72 & 200,15 & 192,39 & 189,02 & 13,03 \\
\hline
\end{tabular}

TABELA 2 - Apresentação do programa do "broken-line" no SAS.

\section{data one;}

input $x y$;

cards;

18159.26

21203.72

24200.15

27192.39

30189.02

;

proc nlin;

parameters $\mathrm{l}=200 \mathrm{u}=-500 \mathrm{v}=10 \mathrm{r}=25$;

$\mathrm{z} 1=(\mathrm{x}<\mathrm{r})^{*}(\mathrm{r}-\mathrm{x})$;

$z 2=(x>r)^{*}(x-r)$;

model $\mathrm{y}=1+\mathrm{u}^{*}(\mathrm{z} 1)+\mathrm{v}^{*}(\mathrm{z} 2)$;

proc nlin;

parameters $\mathrm{I}=200 \mathrm{u}=-\mathbf{5 0 0} \mathrm{r}=\mathbf{2 5}$;

$\mathrm{z} 1=(\mathrm{x}<\mathrm{r})^{*}(\mathrm{r}-\mathrm{x})$;

model $\mathrm{y}=1+\mathrm{u}^{*}(\mathrm{z} 1)$;

run;

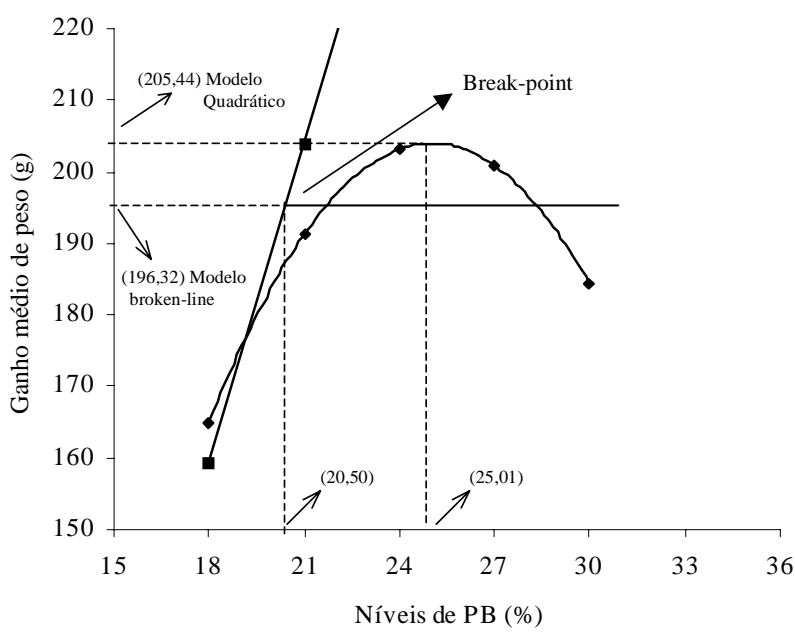

Figura 2 - Aplicação dos modelos regressão segmentada e linear quadrático aos resultados de Vidal et al. (1998). 
TABELA 3 - Apresentação do resultado do "broken-line" no SAS.

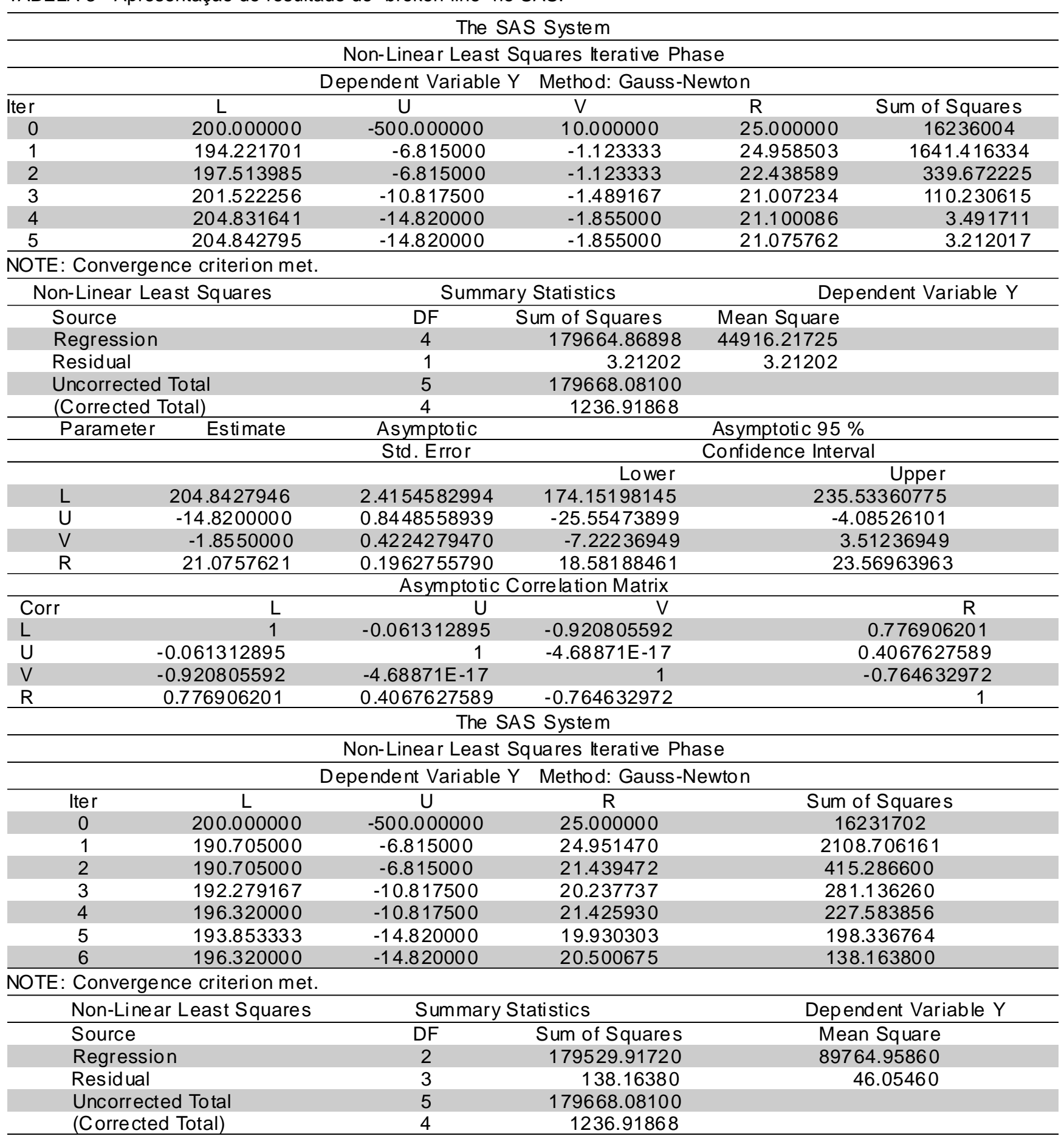

NOTE: The (approximate) Hessian is singular.

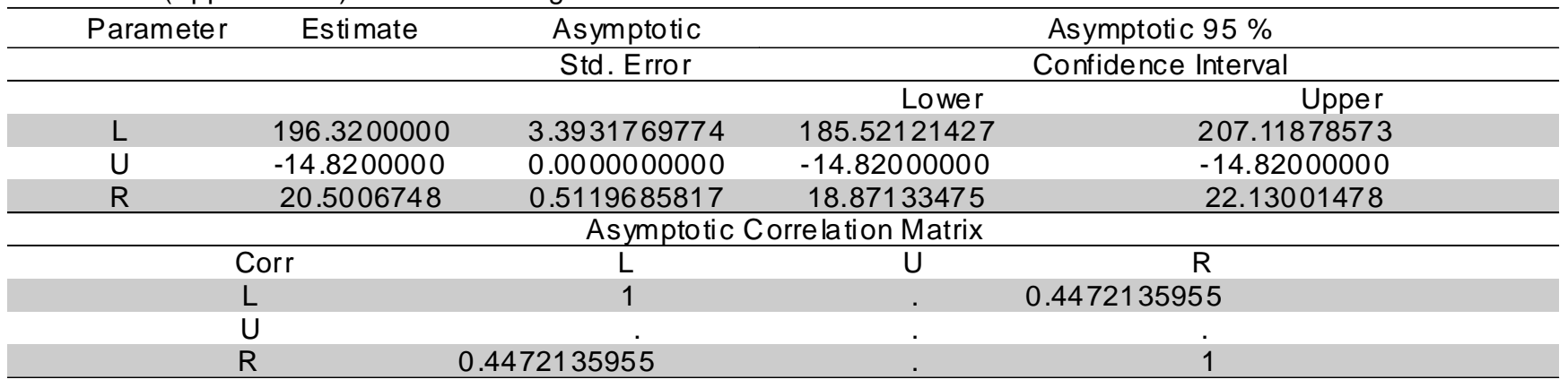




\section{REFERÊNCIAS BIBLIOGRÁFICAS}

ANDERSON, R.J.; KIENHOLZ, E.W.; FLICKINGER, S.A. Protein requeriments of smallmoth bass and largemouth bass. Journal of Nutrition, v.111, p.1085-1097, 1981.

BAKER, D.H.; ALLEN, N.K.; BOOMGAARDT, J.; GRABER, G.; NORTON, H.W. Quantitative aspects of D-and L-tryptofan utilization by the young pig. Journal of Animal Science, v.33, p.42-46, 1971.

CASTAGNOLLI, N. Fundamentos de nutrição de peixes. Piracicaba: Livroceres, 1979. 108p.

CARNEIRO, D.J.; RANTIN, F.T.; DIAS, T.C.R. Níveis de proteína e energia bruta em dietas práticas para crescimento de pacu (Piaractus mesopotamicus) em diferentes temperaturas: I Efeitos sobre o desempenho de produção. In: SIMPÓSIO BRASILEIRO DE AQUICULTURA, 6., Natal, 1990. Resumos. Natal, 1990. p.59.

$\mathrm{CHO}$, C.Y. Feeding for rainbow trout and other salmonids. With reference to current estimates of energy and protein requeriments. Aquaculture, v.100, p.107-123, 1992.

COWEY, C.B. Nutrition: estimating requeriments of rainbow trout. Aquaculture, v.100, p.177-189, 1992.

De LONG, D.C.; HALVER, J.E.; MERTZ, E.T. Nutrition of salmonoid fishes:. VI. Protein requeriments of chinook salmon at two water temperatures. Journal of Nutrition, v.65, p.589-599, 1958.

DUNCAN, P.L.; LOVELL, R.T.; BUTTEWORTH, C.E.; FREEBERG, L.E.; TAMURA, T. Dietary folate requeriment determined for channel catfish, Ictalurus punctatus. Journal of Nutrition, v.123, p.1888-1897, 1993.

EUCLIDES, R. F. Manual de utilização do programa SAEG (Sistema para análises estatísticas e genéticas). Viçosa: UFV, 1983. 59p.

GATLIN, D.M.III Nutrition and feeding of red drum and hybrid striped bass. In: INTERNATIONAL SYMPOSIUM ON ANIMAL AND AQUACULTURE FEEDSTUFFS BY EXTRUSION TECHNOLOGY, 1., Águas de Lindóia, 1998. Proceedings. Campinas: UNICAMP, 1998. p.11.

GRIFFIN, M.E.; WILSON, K.A.; BROWN, P.B. Dietary arginine requeriment of juvenile hybrid striped bass. Journal of Nutrition, v.124, p.888-893, 1994.

KEEMBIYEHETTY; GATLIN III, D.M. Total sulfur amino acid requeriment of juvenile hybrid striped bass (Morone chrysops x M. saxatilis). Aquaculture, v.110, p.331-339, 1993.

KIANG, M. Principles of aquaculture feed production by cooking extrusion. In: INTERNATIONAL SYMPOSIUM ON ANIMAL AND AQUACULTURE FEEDSTUFFSBY EXTRUSIONTECHNOLOGY, 1., Águas de Lindóia, 1998. Proceedings. Campinas: UNICAMP, 1998. p.16.

MAHAJAN, C.L.; AGRAWAL, N.K. Nutritional requeriment of ascorbic acid by indian major carp, Cirrhina mrigala, during early growth. Aquaculture, v.19, p.37-48, 1980.

MAMEESH, M.S.; SCHENDEL, H.E., NORTON, H.W.; JOHNSON, B.C. The effect of penicillin on the thiamine requeriment of the rat. British Journal of Nutrition, v.10, p.23-27, 1956
MERCER, L.P. The quantitative nutrient-response relationship. Journal of Nutrition, v.112, p.550-566, 1982.

MOORE, B.J.; HUNG, S.S.O.; MEDRANO, J.F. Protein requeriment of hachery-produced juvenile white sturgeon (Acipenser transmontanus). Aquaculture, v.71, p.235-245, 1988.

PEZZATO, L.E. O estabelecimento das exigências nutricionais das espécies de peixes cultivadas. In: SIMPÓSIO SOBRE MANEJOE NUTRIÇÃO DE PEIXES, 1., Piracicaba, 1997. Piracicaba: CBNA, 1997. p.45-62.

ROBBINS, K.L.; NORTON, H.W.; BAKER D.H. Estimation of nutrient requeriments from growth data. Journal of Nutrition, v.109, p.1710-1714, 1979.

ROBBINS, K.L. A method, SAS program, and example for fitting the broken-line to growth data. Tennessee: University of Tennessee, Agricultural Experiment Station, 1986. 8p. (Research Report 86/09).

ROBINSON, E.H.; WILSON, R.P.; POE, W.E. Arginine requeriment and apparent absence of a lysine-arginine antagonist in fingerling channel catfish. Journal of Nutrition, v.111, p.46-52, 1981.

SANTIAGO, C.B.; LOVELL, R.T. Amino acid requeriments for growth of nile tilapia. Journal of Nutrition, v.118, p.1540-1546, 1988.

SAS Institute. SAS user's guide: statistics. 5.ed. Cary: Statistical Analysis System Institute, 1985.

SCHULTZ, A.R. Analysis of nutrient-response relationships. Journal of Nutrition, v.117, p.1950-1958, 1987.

SHIAU, S.H.T. Vitamin C requeriment of grass shrimp, Penaeus monodon, as determined with L-ascorbyl-2-monophosphate. Aquaculture, v.122, p.347-357, 1994.

SHIAU, S.; SUEN, G. Estimation of the niacin requeriments for tilapia fed diets containing glucose or dextrin. Journal of Nutrition, v.122, p.2030-2036, 1992.

SHIAU, S.; LIU, J. Quantifying the vitamin K requeriment of juvenile marine shrimp (Penaeus monodon) with menadione. Journal of Nutrition, v.124, p.277-282, 1994.

SHIAU, S.; SUEN, G. The ditary requeriment of juvenile grass shrimp (Penaeus monodon) for niacin. Aquaculture, v.125 p.139-145, 1994.

STEFFENS, W. Principles of fish nutrition. England: John Wiley \& Sons, 1989. 379p.

TACON, A.G.J. Nutritional fish pathology. Rome: FAO, 1992. p.1-75.

VIDAL, M.V.J.; DONZELE, J.L.; CAMARGO, A.C.S.; ANDRADE, D.R.; SANTOS, L.C. Níveis de proteína bruta para tambaqui (Colossoma macropomun), na fase de 30 a 250 gramas: 1 . Desempenho dos tambaquis, Revista da Sociedade Brasileira de Zootecnia, v.27, p.421-426, 1998.

WILSON, R.P. Amino acids and proteins. In: HALVER, J.E. (Ed.) Fish nutrition. London: Academic Press, 1989. p.111-151.

ZEITOUN, I.H.; DULLREY, D.E.; MAGEE, W.T.; GILL, J.L.; BERGEN, W.G. Quantifying nutrient requeriments of fish. Journal of the Fisheries Research Board of Canada, v.33, p.167-172, 1976.

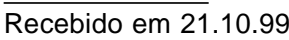

